\title{
Wind Turbine Wake Effect Visualization and LiDAR Measurement Techniques
}

\author{
Michael McKinnon, David A. Johnson \\ Mechanical and Mechatronics Engineering \\ University of Waterloo \\ Waterloo, Canada \\ mmckinno@uwaterloo.ca
}

\begin{abstract}
The expansion of wind energy development has resulted in larger wind farms and closer placement of turbines to utilize the space available. Each turbine produces a wake that affects downstream turbines, which causes issues such as production loss, blade loading, and fluctuating electrical output. In order to minimize this impact, the wake produced by turbines must first be understood. Experimental methods were used in the exploration of the wake effects of wind turbines. Smoke visualization inside a controlled wind facility was used to determine the helical vortex wake distribution behind a $3.3 \mathrm{~m}$ diameter turbine as well as the tip vortex shedding on the blade. Wind Doppler Light Detection and Ranging (LiDAR) measurement devices are used to measure the velocity of aerosols to determine the wind velocity. This technique can be used to measure the airspeed in the wake of a wind turbine. The University of Waterloo Wind Energy Group has a ZephIR z150 LiDAR to use in this study. The LiDAR was verified for accuracy against a cup and vane anemometer. The results determined that the LiDAR was suitable to provide accurate measurements in further turbine wake experiments. The University of Waterloo's facilities provide the advantage to study wake effects under controlled wind conditions as well as under realistic open-air conditions.
\end{abstract}

Keywords-wind energy; wake effects; LiDAR; wind farm layout

\section{INTRODUCTION}

Increasing energy demands and the need for cleaner electricity sources have fueled the development of renewable energy, especially wind energy [1]. More wind farms are being developed with increased density of turbine spacing to utilize areas with high wind conditions. Therefore, wind turbine interaction is becoming a greater concern when developing and operating wind farms.

Wakes are produced by the wind turbines which affect other downstream turbines. This can cause blade loading problems, affect rotor performance, cause fluctuating electrical output, and reduce production [2]. It is important to study the wake effects of wind turbines to be able to design wind farms to reduce the effects on downstream turbines.
Several methods have been explored to measure and simulate the behaviour of the wakes. Some key behaviour characteristics include wake recovery, meandering, and expansion [2]. Experimental methods include wind tunnel tests and field measurements. The University of Waterloo's Wind Energy Group has the capability to test small-scale wind turbines in both wind tunnel and field conditions. The issue with most wind tunnel experiments is obtaining accurate results while scaling wind turbines to fit the wind tunnel specifications. The University of Waterloo's Controlled Wind Facility is able to house a $3 . .3 \mathrm{~m}$ diameter wind turbine with the benefit of obtaining controlled wind conditions up to $13 \mathrm{~m} / \mathrm{s}$. Additionally, the University has a $10 \mathrm{~m}$ wind turbine located in the external environment to test actual field conditions that would be experienced by commercial wind turbines.

With these two wind turbines, the characteristics of wind turbine wakes can be studied and compared to get an understanding of the theoretical and actual behaviour of the wake. Experimental analysis using smoke visualization gives a visual of the wake profile, while a Light Detection and Ranging (LiDAR) measurement device measures the air speed in the wake. Both methods are used to determine the wake effects of the turbines in controlled and external conditions.

\section{EXPERIMENTAL FACILITIES}

Experiments to determine the behaviour of wind turbine wakes were tested inside the University of Waterloo Controlled Wind Facility. The large-scale wind turbine testing facility uses six $75 \mathrm{~kW}$ variable speed fans to obtain consistent wind conditions up to $13 \mathrm{~m} / \mathrm{s}$ in the test area [3]. Fig. 1 shows the

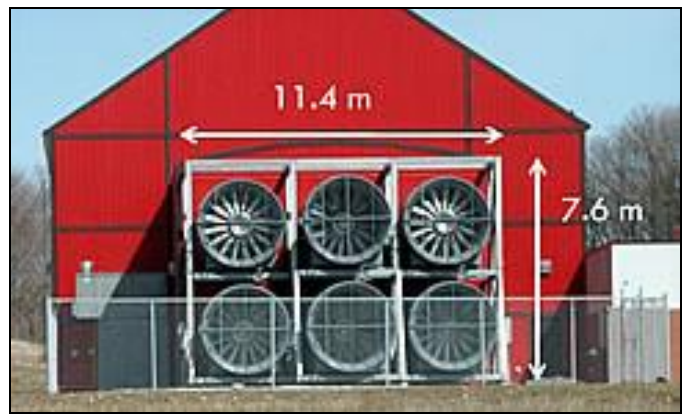

Figure 1. Controlled Wind Facility fans 
fans on the outside of the facility.

A $3.3 \mathrm{~m}$ diameter wind turbine, shown in Fig. 2(a), was developed by the UW Wind Energy Group to operate inside the controlled wind facility. The wind turbine, called the UW WEG turbine, has an output of $3 \mathrm{~kW}$ under $11 \mathrm{~m} / \mathrm{s}$ wind conditions.

A small-scale, grid connected wind turbine is located in the Region of Waterloo and is used by the Wind Energy Group for open-air testing. The turbine, manufactured by Wenvor Technologies, has two blades with passive variable pitching and a diameter of $10 \mathrm{~m}$. The hub height is $30 \mathrm{~m}$ and has a power rating of $35 \mathrm{~kW}$. The Wenvor turbine is shown in Fig. 2(b).

\section{FLOW VISUALIZATION}

Smoke visualization was used inside the Controlled Wind Facility to gain a visual understanding of the wake behaviour as well as provide quantitative measurements. Two smoke visualization techniques exist to study the wake behaviour of turbines [4]. Each method was explored in the experiments. The first method attached smoke emitters to the tip of the one of the blades. This method was used to analyze the wake behind the turbine. The second method ignited smoke in front of the turbine directed at the tip of the blade sweep to analyze the tip vortices.

Both experiments were performed at a wind speed of $6 \mathrm{~m} / \mathrm{s}$ and constant rotational speed of 100 RPM with the UW WEG turbine, resulting in a tip speed ratio of approximately 3.9. The behaviour of the smoke was captured at various views.

A view of the progression from the tip ejection experiment using method 1 can be seen in Fig. 3. As the smoke propagates downstream, a helical pattern can be seen. This shows a typical helical vortex wake distribution. As vortex cylinders are shed from the blades of the turbine, they follow a helical path due to the rotation of the blade. The wake expansion can also be

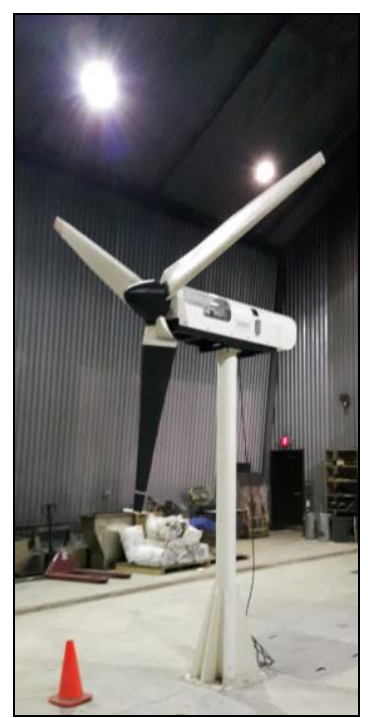

(a)

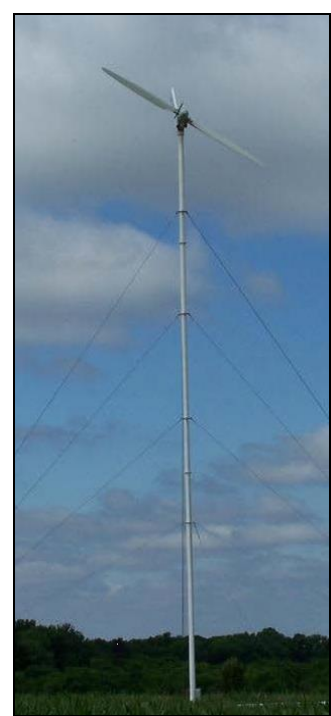

(b)
Figure 2. University of Waterloo's wind turbines: (a) UW WEG Turbine and (b) Wenvor Turbine

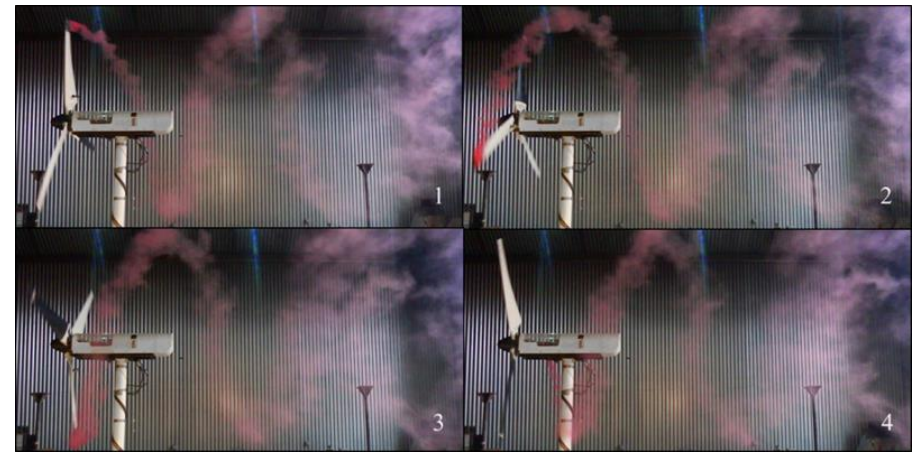

Figure 3. Tip smoke visualization progression of the UW WEG turbine for $6 \mathrm{~m} / \mathrm{s}$ wind speed and a blade rotaion of $100 \mathrm{RPM}$

determined from the smoke visualization experiment. As the smoke moves downstream, the diameter increases due to this expansion.

A close up of the tip of the blade in Fig. 4 shows the angle at which the smoke leaves the blade tip. This is known as the helix angle, $\phi$, or the flow angle at the blade tip [5]. This angle is used to determine the direction of the vortex cylinder parallel to the axis of rotation. As a result, the induced velocity at the rotor plan can be calculated using Biot-Savart law if the vorticity strength in known. The experiment resulted in a helix angle of $25^{\circ}$ from the setup parameters.

The second smoke emission method released smoke upstream of the turbine to visualize the vortices being shed from the blade tips. A tip vortex forms as the blade passes through the smoke. The vortex moves downstream and another is formed as the next blade passes. This effect is seen in the results of the experiment in Fig. 5, which shows a tip vortex being formed and vortex that has moved downstream with the flow. The distance between these vortices is related to the helical path of the vortex cylinders that was seen in the previous experiment.

\section{LIDAR MEASUREMENTS}

Wind Doppler LiDARs have the capability to measure the velocity of particulates in the air, which are used as seeding material to determine the wind speed [6]. The LiDAR achieves

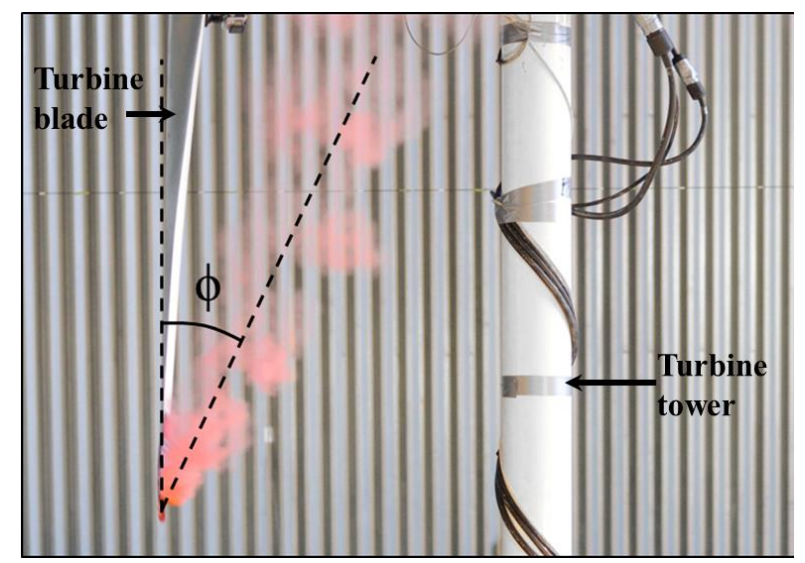

Figure 4. Helix Angle of the UW WEG turbine for $6 \mathrm{~m} / \mathrm{s}$ wind speed and a blade rotaion of $100 \mathrm{RPM}$ 


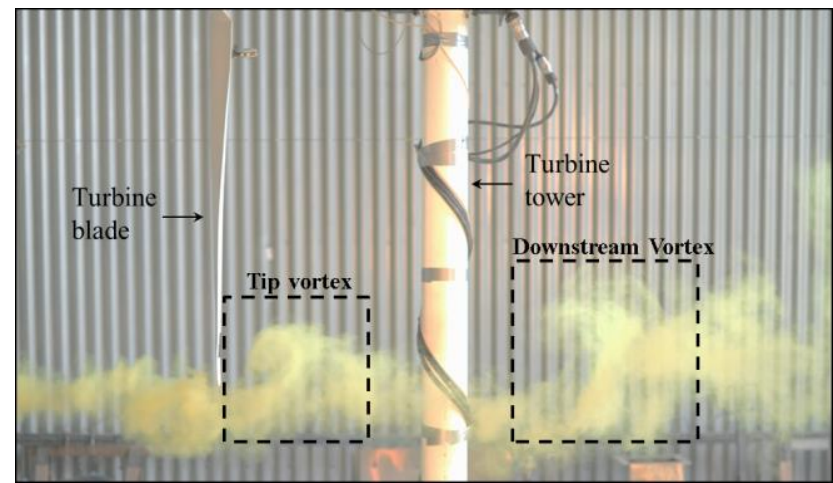

Figure 5. Upstream smoke visualization of the UW WEG turbine $6 \mathrm{~m} / \mathrm{s}$ wind speed and a blade rotaion of 100 RPM

this by the use of a laser, which uses the Doppler Effect from the scattered light reflected from the particulate to calculate the speed of that particle.

Therefore, to measure the velocity of the air in the wake of the turbine, a ZephIR z150 LiDAR, shown in Fig. 6, is used. The LiDAR has the ability to measure various heights and uses a conical sweeping pattern to calculate the direction of the wind. Therefore, it can be used to get a representation of the velocity profile in the wake as it progresses downstream.

Before using the LiDAR for wake measurements, it was first verified for accuracy [8]. A test was conducted to compare the LiDAR against a cup and vane anemometer, each at different heights in atmospheric conditions. The anemometers were attached to the Wenvor wind turbine tower and located at a vertical height of $10 \mathrm{~m}$ from ground level and a height of 20 $\mathrm{m}$ for the cup and vane anemometers respectively.

Due to constraints on the LiDAR, a minimum height of 12 $\mathrm{m}$ was achieved to compare against the cup anemometer. The vane anemometer was used to verify the wind direction. The LiDAR was located $18 \mathrm{~m}$ away from the Wenvor tower to avoid interference due to the conical sweeping of the laser. The LiDAR was placed at a similar distance away from a nearby tree line and ensured that the ground level was consistent with the tower. A view of the setup is shown in Fig. 7. Data was

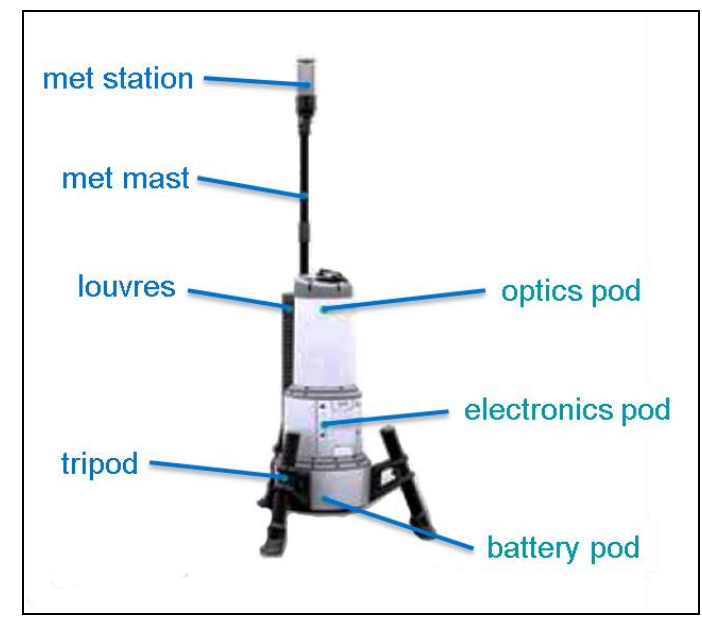

Figure 6. ZephIR z150 LiDAR [7] collected in 10-minute averages over the span of one hour.

The result of the velocity comparison for the LiDAR and cup anemometer is shown in Fig. 8. Due to the limitations of the LiDAR minimum measurable height, the measurements were scaled based on the power law to get equivalent values at $10 \mathrm{~m}$. Both measurements followed the same trend and were within the error of measurements. However, the data was skewed, which could be the result of the scaling or a bias in the cup anemometer measurements.

Similar to the velocity comparison, the direction measurements also showed similar trends as seen in Fig. 9. Again, the comparison shows the measurements were with error. Therefore, it was concluded that the LiDAR measurements were within reasonable accuracy to use for wake measurements.

In order to take measurements in the wake of a turbine, the LiDAR needs to be modified to convert the conical sweep of the laser to a single point line-of-sight laser [9]. This ensures that the measurement position can be placed at a single point and then traversed to gain a profile of the wake.

\section{CONCLUSION}

Understanding wake behaviour of wind turbines is important for the development of new wind farms and design of wind turbines. Understanding can be achieved through experiments using flow visualization and LiDAR measurements.

The University of Waterloo Wind Energy Group uses two wind turbines to contribute to the study of wakes. The two turbines have the benefit to compare controlled wind versus open-air conditions. A controlled wind facility allows for the testing of a $3.3 \mathrm{~m}$ diameter wind turbine under steady wind conditions up to $13 \mathrm{~m} / \mathrm{s}$, while an external $10 \mathrm{~m}$ diameter turbine experiences realistic variable wind effects.

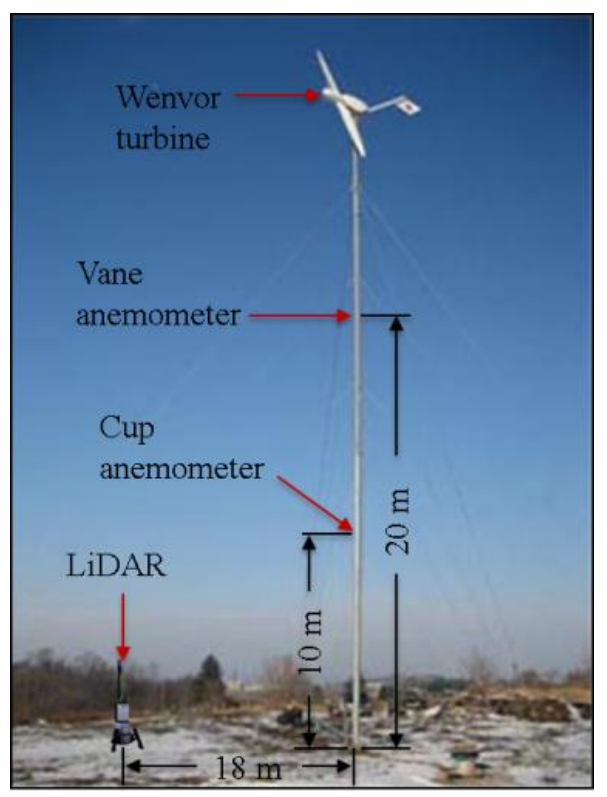

Figure 7. LiDAR verification test setup 


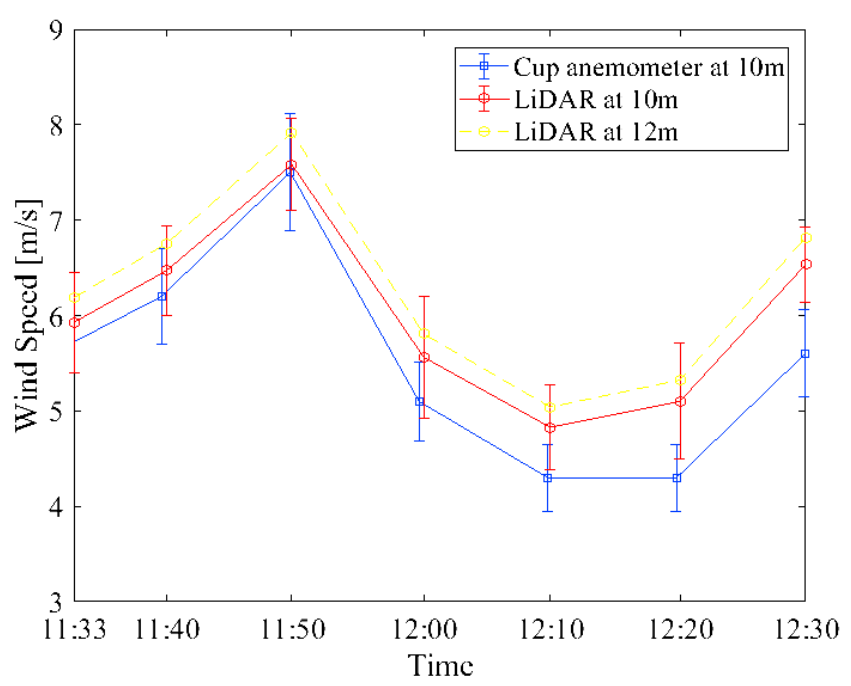

Figure 8. Cup anemometer and LiDAR velocity comparison

Smoke visualization was used to determine the wake characteristics such as a helical vortex distribution, tip vortex shedding, and wake expansion inside the Controlled Wind Facility. This was completed with two smoke visualization techniques that emit smoke from the tip of a turbine blade and another to emit smoke upstream of the turbine directed at the sweeping blade tips. In the first method, the helix angle was able to be measured with a value of $25^{\circ}$. The second method showed the formation and downstream propagation of the tip vortices.

Wind Doppler LiDAR measures the speed of aerosols in the wind to calculate the wind speed. A ZephIR z150 LiDAR was measured against a cup anemometer at a height of $10 \mathrm{~m}$ to verify the wind velocity measurements. The LiDAR was also measured against a vane anemometer with a $20 \mathrm{~m}$ height to verify wind direction measurements. The comparison between the LiDAR and both anemometers showed the same trend and were within error, resulting in the conclusion that the LiDAR had sufficient accuracy to use in the measurement of air speeds. Therefore, the LiDAR is able to be used to measure the

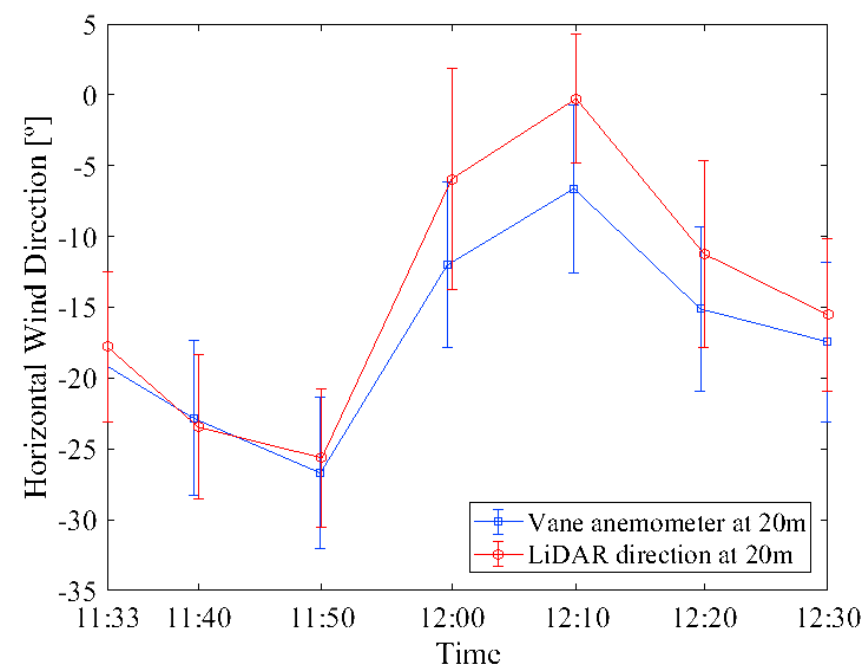

Figure 9. Vane anemometer and LiDAR wind direction comparison velocity profile in the wakes of the turbines.

The comparison between controlled and external conditions will allow for a better relation between the theoretical understanding of wake effects and the actual behaviour of a wind turbine. With this, better optimization of wind farms can lead to increased density while reducing production losses and turbine maintenance.

\section{ACKNOWLEDGMENT}

The authors acknowledge the help and support of the University of Waterloo Wind Energy Group and technicians in the aid of experiments and contribution to the research topic.

\section{REFERENCES}

[1] National Energy Board, "Canada's Energy Future 2017," Governement of Canada, Calgary, 2017.

[2] R. Stevens, and C. Meneveau, "Flow structure and turbulence in wind farms," Annual Review of Fluid Mechanics, vol. 49, pp. 311-339, 2017.

[3] T. E. Gallant, and D. A. Johnson, "In-blade angle of attack measurement and comparison with models," Journal of Physics: Conference Series, 2016.

[4] L. J. Vermeer, J. N. Sørensen, and A. Crespo, "Wind turbine wake aerodynamics," Progress in Aerospace Sciences, vol. 39, pp. 467-510, 2003.

[5] T. Burton, D. Sharpe, N. Jenkins, and E. Bossanyi, Wind Energy Handbook, John Wiley \& Sons Ltd., Chester, 2001.

[6] C. Werner, and C. Weitkamp, Lidar: Ch. 12 Doppler Wind Lidar, Springer Science+Business Media Inc., New York, 2005

[7] Natural Power, "ZephIR Operations and Maintenance Manual," ZephIR LiDAR, Malvern, Worcs, 2009.

[8] M. Cayla, " Comparison of ZephIR measurements against cup anemometry and power curve assessment," Natural Power, Strasbourg, 2010

[9] F. Bingöl, "Adapting a Doppler laser anemometer to wind energy," Master's Thesis, Technical University of Denmark, Lymgby, 2005. 